\title{
The Changing Nature of Writing: Prose or Code in the Classroom
}

\author{
Alan REA \\ Western Michigan University \\ Doug White \\ University of Northern Colorado
}

\begin{abstract}
With the increased use of powerful word-processing programs and with the ease of writing Web pages with hypertext markup language (HTML) and "what you see is what you get" (WYSIWYG) editors, students want to experiment with fonts, colors, images, and sounds. Most of the information students receive outside of the classroom is a multimedia experience (many are from the so-called MTV Generation). Asking them to use print solely for expression goes against how most communication takes place in the world outside academia. Not allowing students to experiment with these new forms of writing hampers their ability to learn effective communication skills.

Our discussion, then, focuses on how instructors can effectively teach and evaluate this "new" writing style. Focusing on teaching HTML scripting and/or Web-page writing, as well as evaluating Web pages according to basic criteria, this discussion maps a pedagogy for hypertext writing. When writing and evaluating in this medium, issues such as audience and purpose, design, and function all remain important. New issues, however, come to the forefront: contextualized hyperlinks, navigability, color schemes, and image, audio, and video integration become a part of the writing task as well. Balancing between effective presentation and sensory overload becomes a daunting task for many students, as well as instructors.
\end{abstract}

Heuristic Hypertext Pedagogy Web Writing World Wide Web Writing Visual Design

Sooner or later most Web users want to become Web writers. Working with electronic text already challenges the fixity of print with the malleability of words on the screen. Moreover, electronic text undermines cultural assumptions. Richard Lanham (1993), in The Electronic Word: Democracy, Technology, and the Arts, discussed the move from print to electronic forms and how "the fixed, authoritative, canonical text, simply explodes into the ether" (p. 31).

However, Web writing further heightens the distinction between our culture's privi-

Direct all correspondence to: Alan Rea, Haworth College of Business, Western Michigan University, Kalamazoo, MI 49008-3821. Email: < alan.rea@wmich.edu>. 
leging of print by stressing the use of graphics and other multimedia applications as equal to, or above, the use of words. Instructors should be aware that asking students to write Web pages is asking them to go against much of their academic training. George Landow (1992), in Hypertext: The Convergence of Contemporary Critical Theory and Technology, argued that "much of our prejudice against the inclusion of visual information in text derives from print technology" (p. 51). The more "valuable" the information in a book, the less it is illustrated. Illustrated children's books give way to elaborately written passages of prose and winding philosophical treatise as part of our maturation process. Instead of asking a student to draw or use illustrations in a writing class to enhance essays, instructors spend class time having students write passages of descriptive prose.

When instructors ask students to create Web pages incorporating graphics, colors, and fonts, they are asking students to subvert the premise on which print literacy is based (the privileging of ideas over iconic representation). This subversion can be problematic for instructors as well; they have been trained to privilege logical argument over graphic presentation.

With pictures and fonts, the glowing hyperlinks on Web pages further subvert print culture's dependence on linearity. The linear progressive argument is familiar to writing instructors. Although the five-paragraph essay may be shunned in college writing classes, instructors still look for strong thesis statements supported by various types of evidence. A student's writing is valued if it logically presents an argument and supports it with valid claims organized into recognizable units of thought. Hypertext, and the ability to link thoughts through hyperlinks, allows associations impossible given the constraints of linear prose:

\footnotetext{
Hypertext, in other words, implements Derrida's call for a new form of hieroglyphic writing that can avoid some of the problems implicit and therefore inevitable in Western writing systems and their printed version. Derrida argues for the inclusion of visual elements in writing as a means of escaping the constraints of linearity. (Landow, 1992, p. 43)
}

With the increased use of powerful word-processing programs and with the ease of writing Web pages with hypertext markup language (HTML) and "what you see is what you get" (WYSIWYG) editors, students want to experiment with fonts, colors, images, and sounds. Most of the information students receive outside of the classroom is a multimedia experience (many are from the so-called MTV Generation). Asking them to use print solely for expression goes against how most communication takes place in the world outside academia. Not allowing students to experiment with these new forms of writing hampers their ability to learn effective communication skills.

Our discussion, then, focuses on how instructors can effectively teach and evaluate this "new" writing style. Focusing on teaching HTML scripting and/or Web-page writing, as well as evaluating Web pages according to basic criteria, this discussion maps a pedagogy for hypertext writing. When writing and evaluating in this medium, issues such as audience and purpose, design, and function all remain important. New issues, however, come to the forefront: contextualized hyperlinks, navigability, color schemes, and image, audio, and video integration become a part of the writing task as well. Balancing between effective presentation and sensory overload becomes a daunting task for many students, as well as instructors. An improved pedagogy must be developed to assist instructors and students approach Web writing in a more structured manner. 


\section{WRITING IN A NEW MEDIUM}

To work effectively within the medium, both instructors and students need to understand the medium itself, because it is not only changing culture, but also the means through which people communicate and share information. Print allowed the dissemination of information to many people (much more than when the only source of information was manuscripts or oral tales), but with today's ever-cheaper and more powerful interactive communications technologies (email and the Web, for example), more people have the potential to express their ideas and to influence others. Instead of the select few having access to the mechanisms for book publishing, broadcast television, or radio, people can take part in the new communication possibilities available through the computer.

This radical shift carries with it a two-fold obligation. Instructors must equip students with the critical and analytical skills needed to analyze the information now being disseminated through a new medium. In addition, instructors need to help students learn how to communicate effectively with the computer (whether it be the word-processing program, desktop publishing software, electronic mail, or one of the many other Internet communication technologies).

All this change still begs the question: Why do writing instructors need to concern themselves with all of this? The answer is quite simple: All of these new communication technologies available through the computer entail writing effectively. For students to be able to participate and excel in academia and the world at large, they need to know how to communicate effectively. A large portion of this entails writing. Although most students in composition classes are not planning on making writing a career, knowing how to communicate in electronic contexts is valuable to any career path they might choose. It always has fallen to writing instructors to give students the needed foundation to communicate and express themselves clearly in their future studies. The remainder of our discussion provides instructors with the basic instruments and information to build a Web-writing pedagogy.

\section{THE WORLD WIDE WEB}

Much like the origin of the Internet, various histories disagree as to when the Web actually came into existence. Many histories place the beginnings of what would be called the World Wide Web in 1991, when Tim Berners-Lee released the Web on CERN (Counseil Europeen pour la Recherche Nucleaire) computers. Although it is true that in 1991 the Web became publicly known, Robert Calliau (1997a), a coauthor of the original Web proposal, set the origin of the Web at 1980 in his "A Little History of the World Wide Web." According to Calliau, when Berners-Lee was consulting for CERN, the European particle physics lab in Geneva in 1980, he wrote a notebook program, "Enquire-WithinUpon-Everything," that allowed "links to be made betwen [sic] arbitrary nodes. Each node had a title, a type, and a list of bidirectional typed links" (online). This sounds very much like the beginnings of applied hypertext.

\section{Hypertext}

Because the Web is based on the concept of hypertext, a short explanation is warranted. The Greek hyper meaning "over, above, concerning" does not really capture the impor- 
tance of hypertext. The original idea for hypertext can be traced to an idea in Vannevar Bush's 1945 Atlantic Monthly article. Bush called for a mechanically linked informationretrieval machine - the Memex - that would help information workers and scholars accommodate the exploding amounts of information (Bush, 1945/1999). Bush knew that effective knowledge building could happen only if scholars were able to access and synthesize information as they needed it. Bush argued that one of the main problems of information storage was categorization and how texts were arranged for retrieval. To work around the strict rules of organization, Bush developed (in theory) a mechanical device (the Memex) that would store all of the information on microfilm and retrieve information according to codes attached to the microfilm information. Moreover, the scholar would be able to annotate and cross-reference information and annotations. The Memex would be a cognitive map of the research process. These associative links are, for all intent and purposes, a hypertext trail.

Twenty years later, Bush's theories informed Ted Nelson's research during his experimentation with methods to organize his thoughts as he wrote a book of philosophy. In "Opening Hypertext: A Memoir," Nelson (1992) discussed how he broke down a "structured complex of thought" he termed a structangle (p. 45) and reconnected it in various ways. Eventually, Nelson's connections of the many structangles resulted in what he termed hypertext. In the 1960s, Nelson came up with an idea for the docuverse, or a vast collection of these hypertexts. For Nelson, the docuverse overcomes many of the inadequacies of information systems in database format and current library systems (vast storehouses for print accessible through electronic catalogs). Nelson's Xanadu project (started in 1985) - the docuverse in action - is creating a global hypertext of all information accessible to anyone. Organized by associative links, Xanadu might resemble something of what the Web has become.

In 1985, Brown University's InterMedia project began to make Xanadu a reality; its impact on the Humanities is discussed by George Landow (1990) in "Changing Texts, Changing Readers: Hypertext in Literary Education, Criticism, and Scholarship." In this landmark article, Landow documented the importance of discoveries students made in an "English Literature from 1700 to the Present" class as they created large hypertext and hypermedia documents using InterMedia. Not only does INTERMEDIA allow students to contribute critical scholarship, the contextual hypertext map creates something greater than the parts put together.

Still, hypertext had not yet truly been introduced to the world at large. Apple released Bill Atkinson's HYPERCARD in 1987 (Calliau, 1997b), but exposed this hypertext only to a small group of people (primarily Macintosh users). However, a historical timeline does not reflect the many overlapping events. Berners-Lee had been working on his interactive notebook program since 1980 and in March of 1989 he submitted "Information Management: A Proposal" with a paper entitled, "Hypertext and CERN" as background information. Robert Calliau joined Berners-Lee as a coauthor and developer. In October 1990, they presented a reformulated proposal and termed the system the World Wide Web. In 1991, the Web was publicly released and in 1992, the first text browser was made available by anonymous FTP, thereby allowing for its distribution (Calliau, 1997a). 


\section{Birth of the Web Browser}

In 1993, Marc Andreessen, then a graduate student at University of Illinois-Chicago, presented his thesis and first alpha version of MOSAIC FOR X: a graphical Web browser for the Internet. In 1994, Andreessen left NCSA (National Center for Supercomputing Applications) and formed the Mosaic Communication Corporation, which is now known as Netscape (Calliau, 1997a). With the release of MosAIC to the public, the Web took the Internet by storm. According to Robert Zakon (1997), in "Hobbes' Internet Timeline v2.5," the Web "proliferate[d] at a 341,634\% annual growth rate of traffic in 1993" and when Netscape went public on the stock market it had the third largest NASDAQ IPO share value ever (online).

The graphical Web browser experienced a phenomenal growth rate. In 1994, the Web edged out TELNET to become the second most popular technology on the Internet (behind FTP) and in April 1995 it passed FTP. It is still the most-used technology on the Internet. What started out as 130 sites in June 1993 is now well over 250,000 and growing (Zakon, 1997).

\section{Explanation of the Web}

What Berners-Lee described in 1991 as the World Wide Web was only the beginning of what has become known as the Web. In its most basic sense, the Web is the name given to a vast collection of information, images, sounds, movies, and other forms of various resources located on various Web servers throughout the world. Moreover, each piece of data has the potential to link to another piece of data anywhere in the world through hypertext links commonly referred to as hyperlinks. A user then can access this data with a simple mouse click or a keystroke. Although the amount of Internet information accessible through the Web grows, it is only a part of the Internet-not the other way around.

\section{Who Runs the Web?}

The Web has no one governing body, either. Even though the vast collection of hyperlinks can lead people to believe that the Web is one large seamless entity, no one is in charge. There is, however, an organization called the World Wide Web Consortium, or W3 Consortium, that is led by Tim Berners-Lee, the founder of the Web. Formed in 1994, the W3's main purpose is to "develop common protocols for the evolution of the World Wide Web" (W3C, 1999). This group is not a Web, Inc. or Big Brother; rather, it is concerned with making sure all clients and helper applications conform to certain standards so that all data can be accessed and used by everyone.

Once users are able to access the data on the Web, they will understand the true dynamics of hypermedia and hypertext (many scholars distinguish between pure text and other media; it is not as necessary as some would have us believe). Many of the explanations contained in this print discussion will not do the Web justice. Moreover, much of what one needs to know about the Web can actually be found, with a little time and patience, on the Web itself. In other words, the Web is a metamedium, or a medium 
that can discuss what it does. The Web contains many sites (specific places on the Internet) that discuss what the Web does and how.

\section{SCRIPTING THE WEB}

With the increased use of the Web for communication comes a need for trained communicators to teach students how the effectively use this medium. However, in many cases, instructors must simultaneously learn these skills with their students. To communicate effectively via the Web requires additional technical and design skills than those needed for word processing and many times instructors have not had this training. The following discussion covers many of the basic technical skills and explores many of the content and design issues that should be considered when writing and evaluating Web pages. With this information, instructors can begin to design a set of hyperheuristics to guide students in their development as Web writers.

\section{Basic HTML}

All Web pages are written in hypertext markup language (HTML). HTML is ASCII text with tags enclosed in greater-than and less-than signs $(<>)$. This ASCII script tells a browser how the Web writer wants the Web page to look. Only a few years ago, a user had to learn HTML to write Web pages. This is no longer the case. With various HTML editors, WYSIWYG editors, and add-on features for word-processing programs, a user can script a simple Web page without ever learning HTML.

For most beginners, a WYSIWYG editor (such as Adobe PAGEMILL, Microsoft FronTPAGE, or Netscape COMPOSER) allows a user to implement basic commands and to see what the Web page will look like as it is written. Word-processing programs, like Microsoft WORD, have HTML add-ons like INTERNET ASSISTANT that convert wordprocessed documents into HTML script. However, all of these programs have limitations for the time being. Eventually, many Web writers will want to know the HTML scripts behind the Web pages they find useful because sections of these scripts can be emulated and learned from.

Ultimately, the best way to learn more about HTML is to look on the Web. There are many sources that can guide a user through HTML script (references to these sites are available at http://unix.cc.wmich.edu/rea/CC/). A user also can view the source code (the HTML that creates a page) by choosing "Document Source" or "Page Source" under the "View" pull-down menu in the browser. Moreover, if users want to "experiment" with the HTML script of the page, they can choose the "source" option in the "Save as" screen under the browser's "File" pull-down menu. Then, there is a local copy (on a disk or hard drive) of the HTML that constructed the page and a Web writer can analyze the script.

When viewing code, a Web writer must remember that HTML, like any language, is a dynamic and evolving means for Web writers to communicate with browsers. In turn, these browsers are the means for others to acquire the Web writer's information in the form of Web pages. However, Web pages look different on various systems and browsers. It depends on many variables, but Web writers cannot control the type of computer used to access their Web page or the type of browser used. Moreover, HTML has gone through various revisions starting at HTML 1.0 and is currently at HTML 4.1. Older browsers 
cannot access many of the new features of HTML 4.x, and there is no way to know who the audience will be or what level of technology will be used. Moreover, the many variations between browser versions sometimes make it challenging to write crossplatform scripts.

Although it is impossible for Web writers to know the entire audience and their technology requirements, just as with prose, Web writers should concern themselves with who their primary audience will be. If the audience is skilled Web writers who can be expected to keep current with the technology, then there is no problem using advanced scripting features. On the other hand, if the audience is new to the Web and their equipment might be older (many schools fall into this category), Web writers might want to keep the Web presentation simple so that all computer systems might access it. A simple guideline is to place the minimum browser version needed to effectively view the Web page directly on the Web page. Moreover, the more complicated a Web page, the more need there might be to write a version accessible through text browsers (or at least older graphical browsers), so all users can have access to information or at least know what they are missing.

\section{EVALUATING WEB SITES}

Students will soon find that accessing information on the Web is not as much of a problem as distinguishing between valuable information and eye candy. Search engine technology is catching up with user demand, but it is still a challenge determining the validity and, ultimately, the usefulness of Web-based information.

Evaluation must also be considered from the perspective of both the organization (in this case, academia) and the individual. Organizations crave a means for assessment of work and products; hence we award grades at the end of a course. Several traditional measures are used in Web-page construction to assess this quality, such as hit counters, cookies, etc., but other more reliable means may be needed to assess the perceptions of end users of the product who are reading, researching, and using the tools produced by the organization. In this light, it may be necessary to introduce traditional, theoretical behavioral constructs that are widely known surrogates for success of information systems. Satisfaction (Doll \& Torkzadeh, 1988), perceived usefulness, and perceived ease of use (Davis, 1989) are all tools that are widely accepted surrogates for success in information systems and might be used for this purpose if empirical study supports their use.

Empirical study aside, the fact remains that the power of the Web to contain a nationally known and respected organization's report next to a less than reputable group's propaganda truly can equalize the distribution of information. However, this equality can also make it difficult for students to determine the validity of the information. Familiar print standards do not appear here. Web sites can be aesthetically pleasing, yet contain no relevant information. On the other hand, a Web writer may have simply converted a groundbreaking thesis from a word processor to a text format and put it on the Web. Although the words are not aesthetically pleasing, the content might be invaluable. However, Web sites most likely fall somewhere in between as the line between content and presentation is a permeable boundary at best.

This challenge comes to the forefront when determining source validity on the Web 
because not everything that looks good is necessarily a reliable source. A well-designed Web page means that the Web writer is good at writing HTML and designing an effective Web page, not that the information on that page is necessarily a reputable source for the students' needs. The following criteria for determining the validity of a Web site's content can function as a part of our Web hyperheuristic. (Although we discuss the application of these criteria in relation to evaluating existing Web content, many of these items can be applied to student writing as well.)

\section{Evaluating Content}

Site Location (URL). Site location can be helpful. If a page claims to be written by an organization, yet does not contain an ".org" in the domain name, then claims should be questioned. If the ".com" designation is part of the site, then business interests may skew the information. In the 1996 presidential elections there were two Bob Dole sites: one official site and one parody site. The only difference in the addresses was an ".org" for the official site and a ".com" for the parody. The sites were designed almost identically, but the content was quite different. An ".edu" designation does not necessarily make the Web writer an academic authority, but a student may be able to verify the writer's credentials by searching the academic site. Ultimately, students should consider the caliber of the information on the entire site if this is a feasible option.

Credentials. If a Web writer is unwilling to supply contact information on the Web page either for the writer or the organization sponsoring the Web site, credibility suffers. At minimum, an email contact address is necessary. Also, links to other sites, writings, etc., done by the Web writer can add to credibility (of course, those would have to be evaluated as well).

If the author is writing as part of an organization, then the organization's credibility needs to be verified. Looking for position statements and organizational goals on the site can help. Also, a student might check whether well-known organizations with similar concerns have any knowledge of the host organization. Ultimately, a student may simply need to call the organizations and ask for more information.

Quality Control/Filters. The distinctions between reputable and not-so-reputable Web sites may not be easy to make. This is especially the case with electronic journals. For example, Kairos has an editorial board of established academics in the computers and composition field, it puts forth review and submission guidelines, and basically follows many of the same procedures set up for scholarly journals. Many other electronic journals do not have the same reputation, yet also have editorial boards, review guidelines, etc. Students cannot determine quality unless they spend the time reading the articles and checking writers' credentials. It is an arduous, yet necessary process.

Intended Audience and Purpose. Most instructors would hope determining audience and purpose on the Web would not be a major issue for students because these topics are covered in class in connection to writing. An instructor would be safe in assuming that a student would not cite children's books about the importance of eating vegetables as part of an argument for eating a balanced diet. The American Medical Association is definitely 
a more reliable source than Golden Book. However, this distinction can become problematic on the Web, as the most informed professional sites are not necessarily the best-designed Web sites.

Timeliness. Although some sources can be dated on the Web-especially if they are discussing facts and figures from previous year's findings-most information changes from month to month. Given the nature of the Web, most sites need to be updated continuously. Even if the basic information on the site does not change, hyperlinks to other sites might. A recently updated Web page assures students that the Web writer at least cares enough to check the site periodically.

Tone, Register, and Bias. Given the ease of Web publication, anyone with access to a computer and a server can distribute his or her ideas and personal philosophies all over the world. Even if a site passes much of the criteria discussed above, a student still must pay attention to the reasonableness and objectivity in a Web site. This is especially true if the Web site is meant to influence users in some manner. Students must look at word choice and manner of presentation, as well as world view, to determine if information is skewed.

Documentation and Hyperlink Cross-References. Finally, a student should consider whether the site has documentation to support its assertions or findings. Then a student must determine if this documentation is reliable and if it can be verified. Is there a list of works cited? Moreover, are there other sources that support this site's findings? Another test of site reliability is to find out if it is referenced with a hyperlink from other reputable sites. A student can determine this by conducting a Web search for the site to find related hyperlink references.

\section{CREATING WEB WRITING}

In addition to evaluating, and then applying, the concepts of content evaluation to Web sites, students and instructors must also consider presentation. In many writing contexts, the use of an approved style guide will suffice. As long as pages are formatted and sources are correctly cited, the student and instructor are able to focus on content alone. This is almost never the case with Web writing. Content and presentation always vie for attention in this medium. In many cases, presentation wins out over content as moving images and colored text are often more compelling for students than grammar and syntax. However, in Web writing both content and presentation are equally important and must share the arena. The task of the Web writer and the instructor is striking a balance between the two. Instructors must concern themselves with the HTML scripting and design of the Web pages in addition to developing content as they help students become Web writers.

\section{Audience and Purpose}

Web writing might seem to put instructors in a conundrum. Does asking students to write Web pages incorporating a mixture of images, sounds, and text go against the prose writing supposed to be the focus of a composition course? We think not. For example, the 
criteria of audience and purpose are still very much a part of writing Web pages. Granted, the question of audience might be difficult for students to determine, given the fact that any one of millions of users could theoretically "hit" their page on the Web. However, just as in writing prose, a Web page designed without a specific audience in mind will lack focus and be less useful than one that targets a specific audience. In most cases, students do not write Web pages only to a class audience but also to a specific audience they have discovered online in a newsgroup or a discussion list. Not only will an online audience give continual feedback on a Web page as it evolves, but such audiences can also provide the student with a specific topic to organize the Web page around.

Purpose is important as well. The existing modes of discourse now used in composition classes can be transferred to Web writing as well. For example, Web pages can inform, persuade, or entertain. Moreover, Web pages can immerse students in a dialectic with their audience (an option not always available in prose). Through email, students can receive commentary on their Web pages from a diverse audience and respond to that audience through email and Web page revisions.

\section{Design and Function}

After determining audience and purpose (and this may be part of a recursive process), design and function become a crucial aspect of Web writing. Instructors can use sections of one of the many style guides available on the Web (URLs are available at http:// unix.cc.wmich.edu/rea/CC/). However, depending on the bias of the style guide author, disagreement may exist on certain issues, such as the number of hyperlinks per page. Some designers more concerned with data presentation argue for one index page full of links to every other page at the site, with no links between the various pages. Other designers opt for shorter pages with many links as opposed to one large page with a few. There is no definitive right or wrong. The following categories are organized around issues to consider in Web page design. Many overlap in concerns and all may not be applicable in any one instance, but they are all useful items to consider when writing and evaluating Web pages.

\section{Hyperlinks}

Meaningful Links. Given the power of hypertext to link passages and sites with a few keystrokes, there always is the temptation to include as many links as possible. However, the coherence of a hypertextual presentation requires that readers know that each link is meaningful and contributes to the overall site. Therefore, Web writers must make sure that all links are contextualized within the passage. Not only should it be clear where the link is going but also what the relationship to the link is. For example, each of the following links lead to the same page ("subversion.html"):

More about hypertext.

More about hypertext and its relation to print culture.

More about hypertext and it subversion of linear prose in print culture. 
The first hyperlink leads a user to something about hypertext; the second is a bit more specific, but the third definitely gives hypertext readers an idea as to where they are going if they choose to follow this hyperlink.

Simple File Names. When writing links to separate pages in a site, Web writers should keep file names simple. Although most Web writers have no control over the domain address (e.g., most personal WMU Web pages are located at http://unix.cc. wmich.edu), they do have control over file names. Because most file names are case sensitive, it is always a good idea to keep file names in all lowercase unless capitalization helps readers with the file name (e.g., "WebWriter.html" is easier to read than "webwriter.html"). Moreover, because one mistake in typing will result in a "file not found" (404) error, file names should be kept descriptive, but short.

Repeated Links. Another important aspect of establishing a reliable context within a Web site is avoiding the temptation to link the same word to the same site repeatedly. Although different sections of the presentation might require a repeated hyperlink to the same site, continually linking the word "subversion" or the phrase "linear prose" to "subversion.html" would be frustrating to the hypertext's audience.

Navigation. Effective contextualization of links greatly helps readers navigate Web sites. Readers new to Web navigation and the demands of hypertext especially need cues as to where they are and where they might go. Many Web writers, such as Patrick Lynch and Sarah Horton (1997) of the Yale Style Guide, recommend using print metaphors, such as indexes and table of contents within Web sites to orient readers. Web writers might also consider putting a link back to their home page or the page that begins the particular presentation on every page within that site. This way, lost readers are more likely to return to the site's organizing page rather than simply leave for another Web destination. Moreover, with the complexity of search engines, one of the site pages might be found without the guiding context needed for a complete understanding. A return hyperlink allows the reader to understand the entire site rather than one page taken out of context.

Most importantly, Web writers should never assume that the reader knows as much about the site as they do. It sounds simple, yet too often a Web writer forgets that the readers have not been present as each hyperlink and passage has been crafted and joined to form a coherent presentation.

Color. HTML gives Web writers the options to change the color of hyperlinks. The default selection is blue for unused links and purple (Netscape NAVIGATOR) or red (Internet EXPLORER) for used hyperlinks. If Web writers feel the need to change hyperlink colors for presentation purposes, they must weigh the aesthetic impact of these color changes with the possible confusion that may result. A reader expecting a blue link and instead getting a green may be confused as to whether the link has been used or not and choose not to follow. Worse yet, the reader might follow and find his or her expectations subverted and leave the site. No matter what the Web writer finally chooses, the hyperlink color scheme should be consistent throughout the entire site. 
Color Schemes. HTML allows Web writers the ability not only to change hyperlink colors but also text colors and background colors. Web writers can use images for backgrounds as well. Most beginning Web writers will go a bit overboard with the opportunity to change every color on the Web page. A dark green background with light green text may look fine on the Web writer's monitor, but all systems display color schemes a bit differently. What may be visible on one system might not be on another. What looks to be a pleasing color scheme through one type of monitor may not be on another.

Background/Text Conflicts. Color schemes can become detrimental to presentation when the background color or image makes it impossible to read the text. In addition, Web writers must consider if they want to account for the possibility of users printing the document. Although there is an option in most browsers to disregard color schemes in favor of the default black text on gray background, most users do not know of this option (available under the "Options" pull-down menu or in "Preferences"). Even a simple Web page with white text on a black background will not show up on paper when a user tries to print it on some systems.

Font sizes and types can be problematic to presentation as well. Many of the newer browsers allow various fonts and sizes. However, a Web writer cannot always assume that a user has the newest browser. The Web writer must decide if including fonts different from the browser-set default is important enough to exclude a significant portion of a potential audience.

\section{Images}

Number. Using images and clip art on Web pages moves students closer to Lanham's (1993) digital rhetoric. Nevertheless, the number of images as well as their file size can greatly inhibit the effectiveness of a Web page. Images can provide wonderful illustrations to support and enhance textual points and they can act as guide markers for navigation and interaction. Arrows, email icons, and scanned photographs are just the beginnings of what a Web writer can do on a Web page. However, just as colors can be overdone, so can images. Just as every hyperlink needs to be contextualized, so does each image.

Size. Although images will not confuse users trying to navigate through the site (unless the images are also hyperlinks), they can overtax the user's system. Images require more time to download than text. Large images (100 KB and above) might take minutes depending on the speed of the user's Internet connection. Web pages that take a long time to download should be identified as such on the hyperlink leading to them. Informed users making the choice to follow a hyperlink even though they know there will be a lengthy download time is more willing to wait than uninformed users. Although it should go without saying, a responsible Web writer makes sure that the wait is worth the time invested.

Placement. Once Web writers have determined the ratio of images to text for a certain page, they must work to incorporate the images effectively. Placing all images flush left or centered is not always the best choice. Images can also be integrated effectively using 
height and width commands that allow the image to be resized to the Web page. However, these sizing commands distort the images depending on the original size. Finding the right combination of height and width may take some experimentation.

\section{Sensory Overload}

As browsers and HTML evolve with new versions, more options become available. Images now can be animated, text can change colors and scroll across the screen, movies and sound are more common, and JavaScript and Java Applets (forms of computer programming embedded in Web pages) can be added to make the Web page truly dynamic. Web users need to learn how much is enough and how much is too much. They must take into account download time, audience, and purpose.

\section{Miscellaneous Items to Consider}

List of Links. Anyone who has navigated (or surfed) the Web has run into many lists of links. Most of the time these are individual compilations of favorite sites or list of sites organized around a topic. These lists can be useful if they contribute to the organization of specific information on the Web. Nevertheless, as with all hyperlinks, contextualizing each link can greatly assist a Web user.

Length of Page. Some Web writers and theorists, such as George Landow (1990, 1992), assert that short passages linked throughout a site take full advantage of hypertext and the Web medium. Pragmatists argue that a large page may take longer to download the first time, but it is less frustrating to navigate and read because the entire page is stored in the browser's cache. Both of these positions are extremes. One or two screenfulls (the amount of information appearing on a standard fifteen-inch screen) per Web page is probably more of the norm. This way, there is a large enough portion to justify a Web page (addressing the pragmatists' concerns) and still ample need for hypertextual linking (addressing Landow's concern). Web writers of a site with a substantial number of links might also consider creating a print version that may take longer to download but can be printed out as a single file if the user so desires.

Outdated Information. Web writers planning on leaving Web pages online for an extended period of time should consider whether they are willing to keep the information on their site current. Otherwise, they might consider removing outdated pages to retain the standards of the site.

Email Address. Web writers should want to hear what people think about their pages. Including an email hyperlink on every page does not guarantee that Web users will write to the Web author, but it does increase the odds. Moreover, many users are more than happy to tell a Web writer that one or more of the hyperlinks suffers from linkrot.

\section{PRACTICAL APPLICATION}

Supplied with the knowledge and the criteria needed for a hyperheuristic, the challenge then becomes classroom implementation. It is valuable for instructors to know just what 
to look for in a Web page, but how do they get the students to apply this information as well? In the concluding sections, we offer a sample form for writers and some pedagogical applications of our discussion the classroom to help students become better Web writers.

We recommend that, at a minimum, instructors bring a presentation system in to show sample Web pages for discussion just as they might do with prose. However, it is also a good idea to get students into a computer classroom to practice these concepts as well.

\section{Critique Web Sites}

Using sites, such as the Cool Site of the Day (http://cool.infi.net), or Vincent Flander's Web Pages that Suck (http://www.websitesthatsuck.com), students can begin to see what other Web users deem to be good or bad. Note, however, that many of these are professionally written and may look good, but fail in other content criteria. This exercise works best if instructors supply students with specific hyperheuristics. Students may also want to look at what other students have done. The Alliance for Computers and Writing site at http://english.ttu.edu/acw/ includes links to student Web sites.

Students can collect three Web sites and evaluate them according to their hyperheuristics. Then, they can post the URLs and the evaluations to the class discussion list. Instructors should encourage students to look at the evaluated sites and discuss each other's evaluation either in class, on the discussion list, or both.

\section{Design a Simple Web Page}

Instructors can choose from one of the shareware HTML editors listed at http://unix.cc. wmich.edu/rea/CC/, or they can use applications available at their institution. In any case, instructors might find it easier to introduce students to Web writing if they use a WYSIWYG editor to avoid using HTML at the onset. Instructors will want to familiarize themselves with the application before bringing it into the classroom as they will, more than likely, become the help desk for students.

After students have spent some time navigating the Web and discussing issues from the hyperheuristic, they should have some idea of how Web pages can look. Instructors should then give each student a disk with a sample Web page template file. This template should consist of basic Web design items, such as bold text, hyperlinks, etc. Using this template, students can then manipulate the various format options, replace text with their own writing, and even insert images they have found on the Web. Moreover, they can begin hyperlinking various sites to their own page, thereby creating a resource for future work.

We have found that conducting this exercise well before students must focus on developing content allows them to experiment with presentation and balance the two entities later in the course.

\section{Create a Web Resource}

This activity is best suited for later in the course. It can be used to take the place of a large individual or group project. Most often, it works well as a substitute for the research paper requirement in many writing classes. Using a hyperheuristic developed for this purpose, instructors should encourage students to not only find all relevant information related to 
their topic on the Web and in the library, but also contextualize this information on a site. After this, students should synthesize and add to the body of knowledge. What can make this truly enticing to students is to have them actively seek input from acknowledged experts in the field via email and USENET postings.

\section{CONCLUSION}

This hyperaccelerated journey into Web writing is but the beginning of what the Web can provide to both students and instructors. The Web is not only a means to acquire data, but also to communicate. Unlike television or radio, which are a one-way street as far as communications go, the Web enables users to talk to one another and actively decide what will be seen and when it will be used. Moreover, the Web allows users to produce information as well. Otherwise, not many would have the chance to produce a television or radio show: The Web is more of an equal-opportunity medium.

Since its beginnings in the early 1990s, the Web has progressed and developed faster than any other technology or communication media to become part of a world culture. There are challenges that still need to be addressed, such as developing standards and providing increased access to students. Nevertheless, Web writing provides a new medium through which students can improve writing and communication skills. The Web is a research tool, a communication technology, and an expressive medium.

However, instructors need to remember that simply having the technology at one's disposal does not automatically make it an effective component of effective writing pedagogies. Instructors need to continue creating and refining guides to make the Web a viable technology of literacy. Ultimately, although the demands of Web writing instruction can be demanding, the benefits far outweigh the costs.

Alan Rea is currently employed as an assistant professor of Business Information Systems. Rea is conducting research in Electronic Communication, Global Information Systems, Visual Rhetoric, and Web-based Training; Rea teaches programming and Web design courses for the Haworth College of Business at Western Michigan University. Rea has published in Kairos and is currently working on designing online courses for WMU.

Doug White worked for Oak Ridge National Laboratories and the Federal Reserve System before returning to academics. Currently, White is conducting research in Discrete Event Simulation Theory, Systems Implementation Issues and Conflict, User Behavior, and International Information Systems. White teaches programming courses for the University of Northern Colorado. White has published in Simulation and Gaming, Information and Management, Simulation Digest, and The Journal of Social Behaviour and Personality.

\section{REFERENCES}

Bush, Vannevar. (1999/1945). As we may think. Atlantic Monthly, 176(1). Available: http:// ccat.sas.upenn.edu/jod/texts/vannevar.bush.html [Accessed May 21, 1997].

Calliau, Robert. (1997a). A little history of the World Wide Web. Available: http://www.w3.org/ History.html [Accessed July 5, 1999]. 
Calliau, Robert. (1997b). Short history of hypertext. Available: http://www.w3.org/History.html [Accessed July 5, 1999].

Davis, Fred D. (1989). Perceived usefulness, perceived ease of use, and user acceptance of information technology. MIS Quarterly, 13(4), 319-340.

Doll, William J., \& Torkzadeh, Gholamreza. (1988). The measurement of end-user computing satisfaction. MIS Quarterly, 12(2), 259-274.

Landow, George P. (1990). Changing texts, changing readers: Hypertext in literary education, criticism, and scholarship. In Bruce Henricksen \& Thais E. Morgan (Eds.), Reorientations: Critical theories and pedagogies (pp. 133-161). Urbana, IL: University of Illinois Press.

Landow, George P. (1992). Hypertext: The convergence of contemporary critical theory and technology. Baltimore, MD: The John Hopkins University Press.

Lanham, Richard A. (1993). The electronic word: Democracy, technology, and the arts. Chicago, IL: University of Chicago Press.

Lynch, Patrick J., \& Horton, Sarah. (1997). Yale style manual. Available: http://info.med.yale.edu/ caim/manual/contents.html [Accessed June 11, 1997].

Nelson, Theodor Holm. (1992). Opening hypertext: A memoir. In Myron Tuman (Ed.), Literacy on-line: The promise (and peril) of reading and writing with computers (pp. 43-57). Pittsburgh, PA: University of Pittsburgh Press.

W3C. (1999). About the World Wide Web Consortium (W3C). Available: http://www.w3.org/pub/ WWW/Consortium [Accessed May 21, 1997].

Zakon, Robert Hobbes. (1997). Hobbes' Internet timeline v2.5. Available: http://info.isoc.org/guest/ zakon/Internet/History/HIT.html [Accessed May 14, 1997]. 